\title{
Low dose tenecteplase restores vessel patency in a clinically relevant porcine animal model
}

\author{
Tseliou E*, Ntalianis A, Diakos N, Pozios I, Repasos V, Katsaros F, Koudoumas D, Dimopoulos S, Tsagalou E, Tsamatsoulis M, Kontopidi \\ V, Margari Z, Terrovitis J and Nanas JN \\ $3^{\text {rd }}$ Cardiology Department, School of Medicine, University of Athens, Athens, Greece
}

\begin{abstract}
Thrombolysis remains the treatment of choice in patients who are far from the catheterization laboratories. Although full dose is advisable low dose has not been preclinically tested. Here we tested the hypothesis that low dose tenecteplase, a recombinant fibrin-specific plasminogen activator can induce efficient thrombolysis even in lower doses than the hitherto indicated, compared to full-dose thrombolytic therapy for ST-segment myocardial infarction. Farm pigs (30-35 kg, $\mathrm{n}=23$ ) were imposed in a clinically relevant model of thrombosis. We used both the common carotid arteries (CA) and the left anterior descending artery (LAD) below the first diagonal. The vessels were initially externally injured (application of pressure by forceps) followed by an appropriate for the vessel dose of thrombin (100 IU for the LAD and 190 IU for CA). Occluding thrombi was achieved in $80 \%$ for the LAD and $57 \%$ for the CA. Ten minutes post occlusion the animals were allocated under continuous electrocardiogram and ultrasonic flow meter monitoring to one of the following groups: Group A) half-dose tenecteplase (2000 IU); Group B) Full dose tenecteplase (5000 IU); Group C) saline only. All animals received 60IU heparin and antiplatelet agents. The follow-up was 120 minutes post therapy administration. Sixty minutes after lytic drug administration recanalization occurred in 58\% of the occluded arteries in Group A, in $85 \%$ in Group B and in $20 \%$ in Group C $(\mathrm{P}=0.028)$. Both tenecteplase regimens were effective for treating carotid artery thrombosis (71\% Group $\mathrm{A}$ vs. $75 \%$ in $\mathrm{Group} \mathrm{B}$ vs. $33 \%$ in $\mathrm{Group} \mathrm{C}, \mathrm{P}=0.286)$, but low dose was significantly less effective in reestablishing flow in proximally occluded LADs (25 \% in Group $\mathrm{A}$ vs. $100 \%$ in $\mathrm{Group} \mathrm{B}$ vs. $0 \%$ in $\mathrm{Group} \mathrm{C}, \mathrm{P}=0.015)$. All vessels with restored flow at 60 minutes remained patent at least for 1 hr. In Group A, at 60 minutes after tenecteplase administration, flow velocity in LAD reached $30 \%$ of the baseline value and $45 \%$ in carotids, while in Group B flow was $160 \%$ and $55 \%$ respectively, suggesting restoration of larger lumen diameter in the treated vessels. Post reperfusion ventricular arrhythmias that required treatment occurred equally in all groups. No differences in hemodynamic tested parameters were observed between the groups. In conclusion, we developed a clinically relevant model useful for testing the efficacy of different thrombolytic regimens. Our data demonstrate that low dose tenecteplase can be successfully used for treating thrombosis of larger diameter vessels, such as the carotids, but appears inadequate for treating $\mathrm{LAD}$ thrombotic occlusions.
\end{abstract}

\section{Introduction}

Myocardial infarction is the leading cause of morbidity and mortality in western countries [1]. The most common cause is rupture of an atherosclerotic plaque with formation of a thrombus leading to occlusion of a coronary artery $[2,3]$. Primary percutaneous coronary intervention (PCI) is routinely performed in the current practice. However, even with advanced commute technological systems catheterization is not always performed timely and valuable time for myocardial salvage is spared.

Thrombolytic reagents emerged as a potential non-operative tool for early in situ recanalization; initial studies reported ante grade perfusion of the infarct related artery in $80 \%$ of the cases within $90 \mathrm{~min}$, resulting in $50 \%$ reduction in mortality $[4,5]$. Additional advantages include wide availability, no need for institutional resources or experienced operator and the ability to deliver them in the emergency department or even before arriving to the hospital [6]. However, early enthusiasm was tempered by suboptimal reperfusion rates, recurrent ischemia and re-infarction and the most important, intracranial or systemic bleeding complications [7].

Among all the fibrinolytic drugs available, tenecteplase is of particular interest since it has the highest fibrin specificity and a long half-life (20-25 $\mathrm{min})$, allowing it to be given as a single-bolus dose [8]. The first clinical trials, ASSENT2 [9] and ASSENT3 [10] proved tenecteplase equally safe and efficient to alteplase, while the latest clinical trial highlighted its role in comparison to PCI $[8,11]$.

Here, we report our experience first with a highly efficient protocol of generating a clinically relevant model of vessel thrombosis and second that low dose of tenecteplase can be efficient in improving flow in large vessels but not in the LAD.

\section{Methods}

\section{Animals}

Experiments were performed on healthy domestic pigs of either sex weighing $30-35 \mathrm{~kg}$ as depicted in Figure 1. The animals were premedicated by intramuscular administration of ketamine $(20 \mathrm{mg} / \mathrm{kg})$ and diazepam $(1 \mathrm{mg} / \mathrm{kg})$. Anesthesia was then induced and maintained by a continuous infusion of sufentanil and pentobarbital into an ear vein. The animal was intubated and ventilated with room air. Body temperature was maintained using a heating pad. Muscle relaxation was achieved by pancuronium bromide infusion $(0.2 \mathrm{mg} / \mathrm{kg})$.

Correspondence to: Eleni Tseliou MD, Department of Therapeutics, Alexandra Hospital, University of Athens, Greece, Tel: 011306976620330; E-mail: etseliou01@gmail.com

Received: December 28, 2015; Accepted: January 14, 2016; Published: January 18,2016 


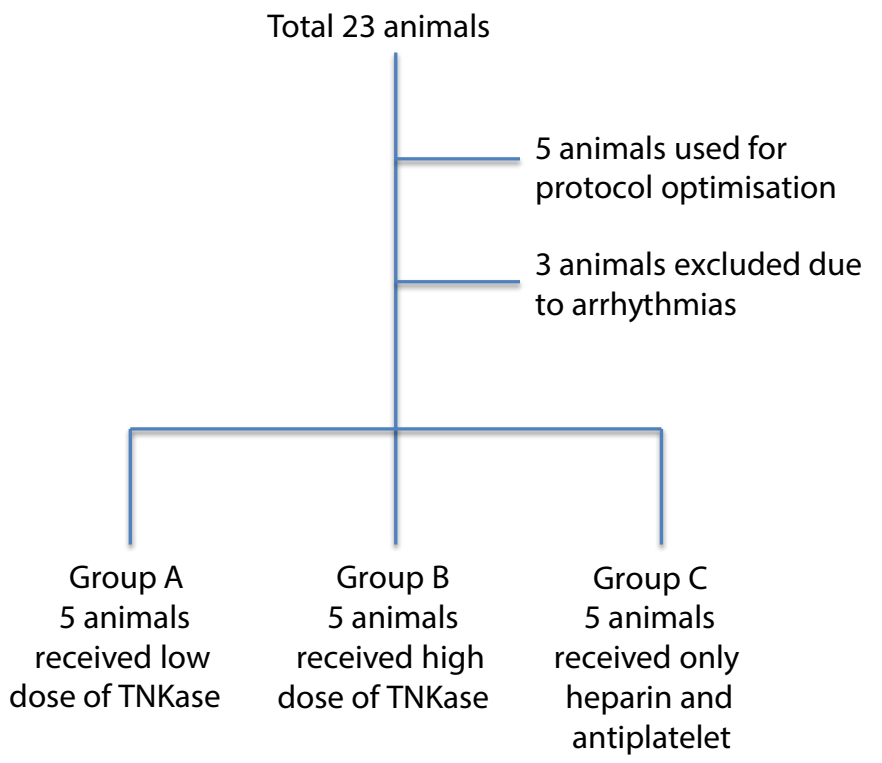

Figure 1. Study design. A total of 23 animals were used for the current study. N=5 were used for the protocol optimisation, $n=3$ were excluded due to arrhythmias and $n=5$ were used in each experimental group. Abbreviation: TNKase=tenecteplase

\section{Experimental procedure}

The right and left cervical area were incised to expose both common carotids (CCA) and the connective tissue around the arteries was dissected carefully. A ring-shaped transit time Doppler flow probe, suitable for the carotid artery diameter $(5-8 \mathrm{~mm})$, was placed proximally around the arteries, and mean blood flow was recorded continuously.

A segment of both common carotids $1 \mathrm{~cm}$ long was isolated distal to the flow probe with two Silastic vessel snares. Intimal injury was then created between the snares by four to five external compressions (one every $8-10 \mathrm{~min}$ ) using hemostat forceps for about 30 minutes. The snares were then tied to occlude the CCA resulting in complete cessation of flow. Thrombin (190 UI) was mixed with blood and injected into the isolated segment. Thirty minutes later, the proximal Silastic snare was removed, immediately followed by the distal snare. Absence of carotid blood flow for at least $10 \mathrm{~min}$ after snare removal was considered as complete vessel thrombosis. A scheme of the experimental protocol and of the thrombosis model is depicted in Figures 2A and 2B.

The same procedure was applied to the left anterior descending artery just below the first diagonal $(2-4 \mathrm{~mm})$. Here a segment $0.5 \mathrm{~cm}$ long was isolated with two Silastic vessel snares carefully, after the first diagonal branch. Intimal injury was then created between the snares by three to four external compressions (one every 10-15 $\mathrm{min}$ ) with a hemostat forceps for about 30 minutes. During this period severe cyanosis was developed in the subtended myocardial area followed hypo-contractility. Thrombin (100 UI) was then injected into the segment and thirty minutes later, the proximal Silastic snare was removed, immediately followed by the distal snare. Absence of coronary blood flow for at least $10 \mathrm{~min}$ after snare removal was considered as complete vessel thrombosis.

\section{Hemodynamic and electrocardiographic measurements}

Continuous electrocardiogram monitoring (ECG; lead II) and a flow meter were used for the evaluation of ST segment changes and recanalization respectively. Frequent Ventricular Premature Beat
(VPB) was defined as more than 5 VPBs per minute. Accelerated Idioventricular Rhythm (AIVR) was defined as a ventricular rhythm with a rate of 60 to 125 beats/minute and frequent episodes of slow ventricular tachycardia. Nonsustained ventricular tachycardia (VT) was defined as 3 or more consecutive ventricular ectopic beats at a rate $>100$ beats/minute and lasting $<30$ seconds. Sustained VT was defined as the last longer than 30 seconds or cause hemodynamic compromise that requires intervention. Ventricular fibrillation was recognized by the presence of irregular nodulations of varying contour and amplitude. A Millar catheter was advanced through an incision at the LV apex in the LV chamber for continuous pressure monitoring. Complete or not complete resolution was defined with respect to initial ECG changes. Re-thrombosis was defined as a new ST-elevation compromised flow compared to the initial evaluation. After surgical preparation the animals were allowed $10 \mathrm{~min}$ for stabilization and then control measurements of ECG, flow and mean arterial pressure (MAP) were taken. Repeat measurements were taken again before occlusion; 10 min post successful thrombosis and before therapy administration; immediately after reperfusion and then every $30 \mathrm{~min}$ until the end of the study. Mean arterial pressure-rate product (PRP) was calculated as $\mathrm{mmHg} /$ beats $/ \mathrm{min} \times 10^{3}$.

\section{Antithrombotic strategy during the procedure}

All animals received antiplatelet and anticoagulation therapy according to the current recommendations. Oral aspirin $300 \mathrm{mg}$ (purchased by Bayern) and oral clopidogrel $600 \mathrm{mg}$ (purchased by Sanofi) at loading doses. Anticoagulation therapy including $60 \mathrm{IU}$ of unfractionated aspirin (purchased by Braun) was given intravenously.

\section{Thrombolytic therapy: dose of administration}

Post successful thrombosis of the vessels animals were allocated to low-dose (Group A) of intravenous tenecteplase (TNK-ase; purchased by Boehringer Ingelheim) slowly administered over 4-5 min through jugular vein catheter, or high dose (Group B) or saline.

\section{Biochemical studies}

In all groups, before euthanasia, blood samples were collected in glass citric tubes (BD Vacutainer) and processed according to manufacturer's instructions. Coagulation markers such as international

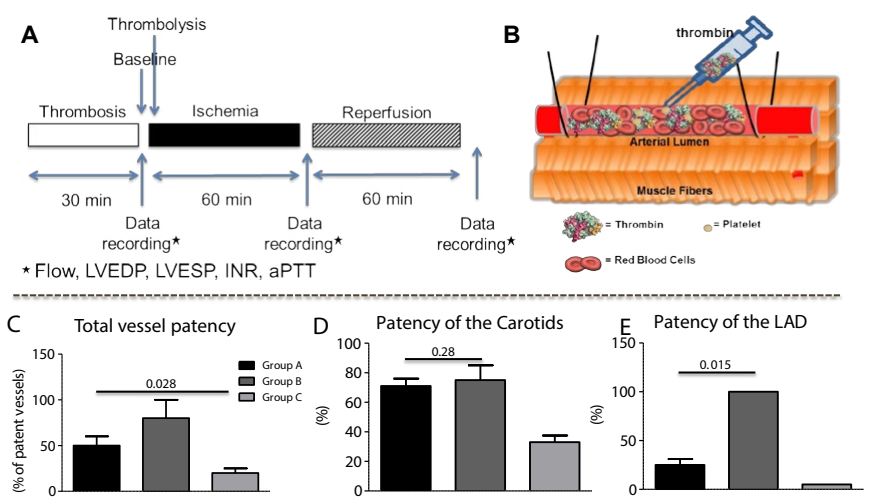

Figure 2. A. Experimental protocol. B. Scheme of the protocol used to induce thrombosis Following external damage to the endothelium, thrombin was injected and total occlusion was validated by a probe distal to the occlusion. C. Approximately $58 \pm 8 \%$ of the vessels in Group A and $85 \pm 15 \%$ in Group B were successfully recanalised compared to $20 \pm 5 \%$ only in Group C. D. No difference between low and high dose Groups was observed as far as carotid patency is concerned. E. Significantly higher number of LADs were recanalised in the high dose of TNKase Group compared to the low dose or the Group that received only heparin. Abbreviation: TNKase=tenecteplase 
normalized ratio (INR), activated partial thromboplastin time (aPTT) and prothrombin time (PT) were evaluated.

\section{Statistical analysis}

The results are expressed as mean \pm SEM, and the data were processed by factorial one-way ANOVA. Individual groups were compared using Dunnett's $t$ test. Differences with $\mathrm{P}<0.05$ were considered statistically significant.

\section{Results}

A total of 23 animals were used in the current study. 5 animals were initially included for the thrombus formation optimization protocol. Another three animals developed ventricular fibrillation during thrombus formation and died before treatment administration. A total of 15 animals were included in the three threated groups.

We decided to use an optimized protocol with thrombin infusion to interrupt vessel flow rather than mechanical interventions with percutaneous catheters so as to better simulate a clinical acute MI scenario. The latter entails a ruptured plaque, adhesion of platelets and thrombi formation. Endothelial disruption by external compressions and infusion of blood with thrombin mimicked this pathology. Results show that successful thrombosis was achieved in $80 \%$ of the carotids and in $57 \%$ of the LAD vessels used.

\section{Recanalization}

Ten minutes after the induction of occlusive thrombi, we tested whether the low or the high dose of TNK-ase are able to restore flow in the thrombosed vessels. To this end, 2000 IU TNK-ase (Group A, n=5) or 5000 IU TNK-ase (Group B, $n=5$ ) or saline only (Group C, $n=5$ ), were injected via the internal jugular vein. All animals received $60 \mathrm{IU} /$ $\mathrm{kg}$ heparin and antiplatelet therapy. No adverse events were observed during the infusion in any of the animals studied. At 60 minutes post lytic drug administration, recanalization occurred in $58 \pm 8 \%$ of the occluded arteries in Group A, in $85 \pm 15 \%$ in Group B and in $20 \pm$ $5 \%$ in Group C (Figure 2C; $\mathrm{p}=0.028$ ). Both tenecteplase regimens were effective for treating carotid artery thrombosis (Figure 2D; $71 \pm 5 \%$ Group A vs. $75 \pm 10 \%$ in Group B vs. $33 \pm 4.5 \%$ in Group C, $\mathrm{P}=0.286$ ), but low dose was significantly less effective in reestablishing flow in proximally occluded LADs $(25 \pm 6 \%$ in Group A vs. $100 \%$ in Group B vs. $0 \%$ in Group C, Figure 2E; $\mathrm{p}=0.015$ ). All vessels with restored flow at 60 minutes remained patent at least for $1 \mathrm{hr}$. In Group A, at 60 minutes after tenecteplase administration, flow velocity in LAD reached $30 \pm$ $22 \%$ of the baseline value and $45 \pm 12 \%$ in carotids, while in Group B $160 \pm 42 \%$ and $55 \pm 18 \%$ respectively (Figure $3 \mathrm{~A}, \mathrm{~B} ; \mathrm{p}=0.028$ ).

\section{Post-Reperfusion Arrhythmias}

ECG monitoring was performed during the whole experimental procedures for arrhythmias detection. No significant differences were observed in the arrhythmias observed during the vessel thrombosis. Previous studies showed that during the first 30 seconds of reperfusion there is a marked inhomogeneity in the action potentials within the ischemic area and at the border that contributes to the occurrence of fibrillation [12]. In line with these data we observed episodes of fibrillation in total 2 out of the 5 animals that required resuscitation within 30 min post reperfusion in Group A and in 4 animals in Group B. Episodes of AIVR were observed in Group C ( 2 out of 5 animals), but not in Group A or B. No statistically significant difference between the groups in terms of sustained ventricular tachycardia, nonsustained ventricular tachycardia, frequent ventricular premature beats, and

\section{A Percent of flow restoration in Carotids}

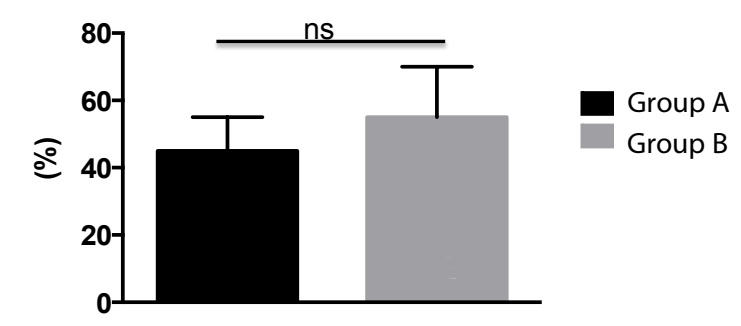

\section{B Percent of flow restoration in the LAD}

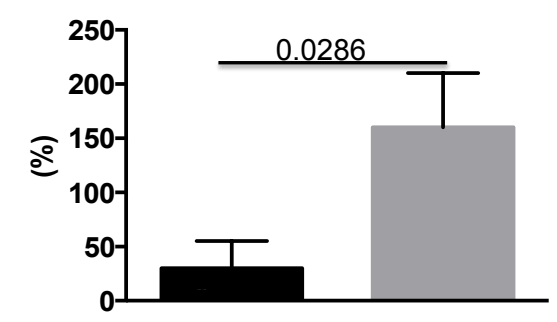

Figure 3. A. Percent of flow restoration in Carotids post high or low dose of TNKase. B. Percent of flow restoration in the LAD post high or low dose of TNKase. Abbreviation: TNKase $=$ tenecteplase

atrioventricular block was found. The association of ST segment regression and reperfusion arrhythmias was also evaluated. The animals in Group B revealed greater ST segment regression and consequently higher rates of reperfusion arrhythmias. The latter may be related to maximal heterogeneity when irreversibly and reversibly injured cells are juxtaposed.

\section{Hemodynamics}

Table 1 summarizes these data. Continuous LV pressure monitoring was performed with a Millar pressure catheter installed in LV apex. There was no statistically significant difference between LV systolic and diastolic pressure between all groups before treatment administration. Systolic blood pressure was significantly compromised in Group C compared to the other 2 groups. Failure to recanalize the LAD may explain this finding.

\section{Biochemical indexes}

No differences were observed in the INR values at the time point of evaluation $(<1.2$ in all animals evaluated) or aPTT or PT. Perhaps the short time post infusion of tenecteplase and heparin that these indexes were evaluated did not interfere with the systemic levels. Nevertheless, our findings are in line with previous reports were concomitant administration of thrombolytics and heparin did not influence acutely the hemostatic pathway $[10,13,14]$.

\section{Discussion}

Restoration of epicardial coronary flow post-acute MI improves clinical outcome [15]. Recanalization can be achieved by means of thrombolysis or primary angioplasty. Although angioplasty is preferred by European and American guidelines accumulated studies question the superiority of angioplasty over thrombolysis $[16,17]$. The safety and the efficacy of tenecteplase has been repeatedly evaluated in clinical trials [8], however the efficacy of low dose of tenecteplase in restoring blood flow has never been tested before. In the current study we focused 
Table 1. Hemodynamics during thrombosis formation experiements.

\begin{tabular}{|c|c|c|c|c|c|c|c|c|c|}
\hline \multirow[t]{2}{*}{ Parameters } & \multicolumn{3}{|c|}{ Heart Rate (beats/min) } & \multicolumn{3}{|c|}{ Mean Arterial Pressure (mmHg) } & \multicolumn{3}{|c|}{ Mean Left Atrial Pressure (mmHg) } \\
\hline & GroupA & GroupB & GroupC & GroupA & Group B & Group C & Group A & Group B & Group C \\
\hline Baseline & $95 \pm 4$ & $101 \pm 2.3$ & $96 \pm 12$ & $98 \pm 1.6$ & $101 \pm 3.2$ & $95 \pm 2.8$ & $4.8 \pm 1.8$ & $4.5 \pm 2$ & $5 \pm 1$ \\
\hline \multicolumn{10}{|l|}{ Reperfusion } \\
\hline $15 \mathrm{~min}$ & $105 \pm 1.9$ & $110 \pm 4$ & $101 \pm 3$ & $95 \pm 3$ & $95 \pm 8$ & $100 \pm 6$ & $9 \pm 2$ & $7.5 \pm 2$ & $9 \pm 3$ \\
\hline $30 \mathrm{~min}$ & $101 \pm 5$ & $112 \pm 8$ & $100 \pm 2.9$ & $100 \pm 8$ & $100 \pm 5$ & $95 \pm 5$ & $8.5 \pm 2$ & $8.5 \pm 3$ & $8.5 \pm 2$ \\
\hline $45 \mathrm{~min}$ & $112 \pm 8$ & $125 \pm 5.8$ & $99 \pm 4$ & $105 \pm 5$ & $110 \pm 4$ & $86 \pm 5$ & $8.5 \pm 2$ & $8 \pm 4$ & $9 \pm 4$ \\
\hline $60 \mathrm{~min}$ & $120 \pm 4.9$ & $135 \pm 4$ & $100 \pm 4$ & $100 \pm 4$ & $110 \pm 4$ & $80 \pm 4$ & $9 \pm 2$ & $8 \pm 4$ & $10 \pm 4$ \\
\hline $75 \mathrm{~min}$ & $120 \pm 6$ & $135 \pm 8$ & $105 \pm 3$ & $110 \pm 5$ & $110 \pm 4$ & $85 \pm 8$ & $8.5 \pm 2$ & $7.5 \pm 2$ & $9.5 \pm 3$ \\
\hline $90 \mathrm{~min}$ & $125 \pm 4$ & $130 \pm 3$ & $103 \pm 4$ & $105 \pm 5$ & $115 \pm 3$ & $85 \pm 5$ & $8 \pm 2$ & $7.5 \pm 2$ & $10 \pm 3$ \\
\hline $105 \mathrm{~min}$ & $125 \pm 5$ & $132 \pm 3$ & $110 \pm 3$ & $105 \pm 5$ & $115 \pm 3$ & $95 \pm 7$ & $8.5 \pm 2$ & $8 \pm 2$ & $11 \pm 2$ \\
\hline $120 \mathrm{~min}$ & $130 \pm 8$ & $135 \pm 3$ & $105 \pm 5$ & $110 \pm 7$ & $110 \pm 5$ & $90 \pm 5$ & $8 \pm 2$ & $8 \pm 2$ & $10 \pm 4$ \\
\hline
\end{tabular}

on thrombolysis and created an experimental model of thrombosis that simulates the clinical pathology of acute MI. Following successful vessel thrombosis we tested the efficacy of low dose of tenecteplase in restoring blood flow in occluded vessels. We demonstrated for a fist time that the low dose of tenecteplase can restore blood flow within one hour of symptom initiation in large vessels such as carotids $(5-8 \mathrm{~mm}$ diameter) but does not suffice to restore flow in the smaller in diameter vessels such as the LAD (2-4 mm).

The most common cause of MI is rupture of an atherosclerotic plaque with formation of a thrombus leading to occlusion of a coronary artery $[2,3]$. Serial in vitro experiments have examined the mechanism of atherosclerosis and have paved the way for new therapeutic drug development [18]. In addition, in vivo experiments in small genetically modified animals have also elucidated the pathology of the coronary syndrome [19]. However, the most suitable model should simulate the human disease. In the current study, we used a swine model to create thrombosis and mimic a clinical scenario of myocardial infarction. We first caused external damage to the common carotids and to the LAD so as to activate the aggregation of platelets and we consequently infused thrombin so as to create thrombosis. Platelet function is similar between swine and humans and this made them suitable for the current protocol [20]. In addition, coronary anatomy in swine is similar to the human except for left azygos vein which drains to the left coronary sinus. These data support the idea of a preclinical model of thrombosis in swine that would enable testing new therapeutic drugs for atherosclerosis. Indeed, following the protocol described in the current study we successfully occluded $80 \%$ of the LADs and $57 \%$ of the common carotids and then we tested tenecteplase efficacy to restore blood flow.

Different thrombolytic drugs have been used in preclinical and clinical studies for acute myocardial infarction with salutary effects reported. Among others tenecteplase is more promising given its high fibrin specificity that enables delivery by single bolus intravenous injection [21]. More specifically, tenecteplase is a genetically engineered plasminogen activator with reduced risk for causing major bleeds due to restricted lytic activity to the plasmin on the fibrin surface that avoids the breakdown of fibrinogen, factor V, factor VIII and a2-antiplasmin [22]. Compared to other thrombolytic regimens, tenecteplase is not affected by other drugs such as nitrates, it has more robust anti-platelet effects and exerts higher thrombolytic potency even when given more than 4 hours after the onset of symptoms $[9,21,23]$. More than 25000 patients have been so far recruited in clinical trials that used tenecteplase as a potential therapeutic regimen making the use of this drug evidence based. In line with previous data we did not observe any changes in the biochemical indexes evaluated in the current study post either low or high dose of tenecteplase without changing the dose of heparin used in parallel [24]. However, low dose of tenecteplase restored blood flow in $25 \%$ of the LADs compared to $100 \%$ restored post full dose. Similar efficacy was observed between the two doses in regards to common carotid blood flow restoration, suggesting that low dose can be used safely for restoration of flow in large arterial vessels but not in the smaller in diameter ones. The latter is important for ischemic strokes where the combination of thrombolysis and interventional techniques is the currently preferable treatment [25].

Our data are in line with the latest clinical trial that used high dose of tenecteplase for vessel recanalization [10]. However, the low dose that was used in patients above 75 years of age was also able to achieve vessel patency in contrast to our findings. A possible explanation may be the smaller diameter of the LAD in our experimental model compared to the human atherosclerotic ones. More specifically, the diameter of the LAD used here, was approximately $2-3 \mathrm{~mm}$ while in humans it reaches $4-5 \mathrm{~mm}$; the latter is closer to the swine carotid arteries that were recanalized with both low and high doses $(\sim 5 \mathrm{~mm})$. In addition, although we used an experimental model of thrombus formation in [14] order to simulate clinical atherosclerotic plaques, the external damage of the LAD can also result in vessel spasm and vessel internal diameter stenosis that may further aggravate the occlusion, requiring higher doses of tenecteplase.

In conclusion, in the current study we successfully created a preclinical model of coronary thrombosis where different regimens can be routinely evaluated. Second, we used this model to test the efficacy of low and high dose of tenecteplase to restore blood flow in the coronaries and the common carotids. We found significant increase in the number of LADs recanalized post full dose of tenecteplase compared to the LADs treated with low dose while in common carotids both regimen doses were equally efficient. Following pioneering clinical trials $[11,26]$ where thrombolysis by means of tenecteplase was very competitive with primary angioplasty when delivered very rapidly, possibly in the pre-hospital phase our data support that tenecteplase may offer a very simple and effective treatment, particularly if subsequent primary angioplasty is offered to the patients with high clinical risk or with recurrent ischemia.

\section{References}

1. Yeh RW, Sidney S, Chandra M, Sorel M, Selby JV, et al. (2010) Population trends in the incidence and outcomes of acute myocardial infarction. $N$ Engl J Med 362: 2155 2165.[Crossref]

2. Fishbein MC, Meerbaum S, Rit J, Lando U, Kanmatsuse K, Mercier JC, et al (1981) Early phase acute myocardial infarct size quantification: validation of the 
triphenyltetrazolium chloride tissue enzyme staining technique. Am Heart $J$ 101: 593600. [Crossref]

3. Davies MJ (1997) The composition of coronary-artery plaques. $N$ Engl J Med 336: 1312-1314. [Crossref]

4. Effectiveness of intravenous thrombolytic treatment in acute myocardial infarction. GruppoItaliano per lo Studio della Streptochinasinell' Infarto Miocardico (GISSI). Lancet 1: 397-402. [Crossref]

5. (1993) An international randomized trial comparing four thrombolytic strategies for acute myocardial infarction. The GUSTO investigators. $N$ Engl J Med 329: 673-682. [Crossref]

6. Armstrong PW, Collen D, Antman E (2003) Fibrinolysis for acute myocardia infarction: the future is here and now. Circulation 107: 2533-2537. [Crossref]

7. Grines CL, Serruys P, O'Neill WW (2003) Fibrinolytic therapy: is it a treatment of the past? Circulation 107: 2538-2542.[Crossref]

8. Logallo N, Kvistad CE, Thomassen L (2015) Therapeutic Potential of Tenecteplase in the Management of Acute Ischemic Stroke. CNS Drugs 29: 811-818. [Crossref]

9. Serebruany VL, Malinin AI, Callahan KP, Binbrek A, Van De Werf F, et al. (2003) Effect of tenecteplase versus alteplase on platelets during the first 3 hours of treatment for acute myocardial infarction: the Assessment of the Safety and Efficacy of a New Thrombolytic Agent (ASSENT-2) platelet substudy. Am Heart $J$ 145: 636-642. [Crossref]

10. Assessment of the Safety and Efficacy of a New Thrombolytic Regimen (ASSENT)-3 Investigators (2001) Efficacy and safety of tenecteplase in combination with enoxaparin, abciximab, or unfractionated heparin: the ASSENT-3 randomised trial in acute myocardial infarction. Lancet 358: 605-613. [Crossref]

11. Armstrong PW, Committee WS (2006) A comparison of pharmacologic therapy with without timely coronary intervention vs. primary percutaneous intervention early after ST-elevation myocardial infarction: the WEST (Which Early ST-elevation myocardial infarction Therapy) study. Eur Heart J 27: 1530-1538. [Crossref]

12. Cascio WE (2001) Myocardial ischemia: what factors determine arrhythmogenesis? $J$ Cardiovasc Electrophysiol 12: 726-729. [Crossref]

13. Antman EM, Louwerenburg HW, Baars HF, Wesdorp JC, Hamer B, et al. (2002) Enoxaparin as adjunctive antithrombin therapy for ST-elevation myocardial infarction: results of the ENTIRE-Thrombolysis in Myocardial Infarction (TIMI) 23 Trial. Circulation 105: 1642-1649.[Crossref]

14. Giugliano RP, Roe MT, Harrington RA, Gibson CM, Zeymer U, et al. (2003) Combination reperfusion therapy with eptifibatide and reduced-dose tenecteplase for
ST-elevation myocardial infarction: results of the integrilin and tenecteplase in acute myocardial infarction (INTEGRITI) Phase II Angiographic Trial. J Am Coll Cardiol 41:1251-1260. [Crossref]

15. Armstrong PW, Collen D (2001) Fibrinolysis for acute myocardial infarction: current status and new horizons for pharmacological reperfusion, part 2. Circulation 103 2987-2992. [Crossref]

16. Armstrong PW (2003) Primary angioplasty or thrombolysis for acute myocardial infarction? Lancet 361: 966; author reply 967-968.

17. DeMaria AN (2008) Lies, damned lies, and statistics. J Am Coll Cardiol 52: 14301431. [Crossref]

18. Kloner RA, Braunwald E (1980) Observations on experimental myocardial ischaemia Cardiovasc Res 14: 371-395. [Crossref]

19. Svenson KL, Bogue MA, Peters LL (2003) Invited review: Identifying new mouse models of cardiovascular disease: a review of high-throughput screens of mutagenized and inbred strains. J Appl Physiol 94: 1650-1659.[Crossref]

20. Bodary PF, Eitzman DT (2009) Animal models of thrombosis. Curr Opin Hematol 16 : 342-346. [Crossref]

21. Modi NB, Eppler S, Breed J, Cannon CP, Braunwald E, et al. (1998) Pharmacokinetics of a slower clearing tissue plasminogen activator variant, TNK-tPA, in patients with acute myocardial infarction. Thromb Haemost 79: 134-139.[Crossref]

22. Tsikouris JP, Tsikouris AP (2001) A review of available fibrin-specific thrombolytic agents used in acute myocardial infarction. Pharmacotherapy 21: 207-217. [Crossref]

23. Collen D, Stassen JM, Yasuda T, Refino C, Paoni N, et al. (1994) Comparative thrombolytic properties of tissue-type plasminogen activator and of a plasminogen activator inhibitor-1-resistant glycosylation variant, in a combined arterial and venous thrombosis model in the dog. Thromb Haemost 72: 98-104. [Crossref]

24. Cannon CP, Gibson CM, McCabe CH, Adgey AA, Schweiger MJ, et al. (1998) TNKtissue plasminogen activator compared with front-loaded alteplase in acute myocardial infarction: results of the TIMI 10B trial. Thrombolysis in Myocardial Infarction (TIMI) 10B Investigators. Circulation 98: 2805-2814. [Crossref]

25. Marshall RS (2015) Progress in Intravenous Thrombolytic Therapy for Acute Stroke. JAMA Neurol 72: 928-934. [Crossref]

26. Bonnefoy E, Lapostolle F, Leizorovicz A, Steg G, McFadden EP, et al. (2002) Primary angioplasty versus prehospital fibrinolysis in acute myocardial infarction: a randomised study. Lancet 360: 825-829. [Crossref]

Copyright: (C2015 El-Ansary D. This is an open-access article distributed under the terms of the Creative Commons Attribution License, which permits unrestricted use, distribution, and reproduction in any medium, provided the original author and source are credited. 